\section{Measuring the growth rate of cells, one at a time}

\author{
Gilles Charvin
}

\begin{abstract}
Silicon-based microscales allow real-time measurements of the growth rate of single cells and open up interesting perspectives for the study of mass biogenesis at the cellular level.
\end{abstract}

Cell mass increase results from all biogenesis processes (production of proteins, lipids, nucleic acids and other molecules). In contrast to specific molecular processes, which can be monitored in single cells using the many fluorescence-based microscopy techniques available, it is difficult to reliably measure the overall mass of single cells and, a fortiori, to determine their growth rate. In this issue of Nature Methods, Godin et al. ${ }^{1}$ report the use of a microfabricated resonator to accurately measure the growth rate of trapped individual live cells (bacteria, yeast and mammalian cells) with high temporal resolution (Fig. 1). Combining this technique with standard genetics and cell biology approaches will undoubtedly provide new insights into the mechanisms controlling cell growth.

First-year college students in biology commonly observe that when cells are grown in appropriate conditions, cell number increases exponentially with time. Obviously, the reason for this is that the cell division cycle is a multiplicative process. As a result, when tracked over several generations, the total biomass of a cell population increases exponentially. But does this exponential mass increase still hold at the level of a single cell and within one cell generation?

It has long been debated whether biogenesis of cellular components results in a constant or, alternatively, cell size-dependent rate of mass increase — or even more complicated: a cell division cycle-dependent increase in mass. Following the simplistic 'more ribosomes produce more ribosomes' rule, one would expect that cellular biomass increases exponentially with time. In budding yeast, this hypothesis is actually supported by several observations that protein content increases exponentially within one cell cycle ${ }^{2,3}$. However, a recent study pointed

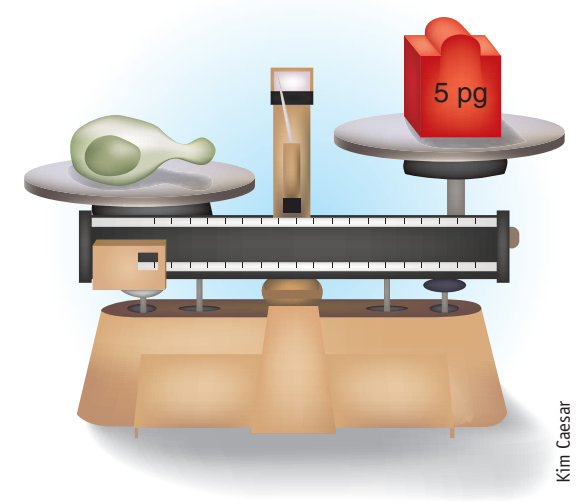

Figure 1 | A microscopic scale can be used to measure variations in the mass of individual cells spanning the range from 100 femtograms to 100 picograms.

out that cell growth rate could be substantially modulated over the course of one division cycle . $^{4}$.

To alleviate this controversy, Godin et al. ${ }^{1}$ developed a technique that allows them to monitor the evolution of the mass of a single cell over time in a suspended microchannel resonator. This silicon-based device is composed of a micrometer-scale cantilever whose resonance frequency is directly related to its mass. Flowing individual cells through a small flow channel that is etched in the resonator results in a slight change in the resonance frequency $^{5}$. From this shift, the buoyancy mass (that is, roughly, the dry mass) of the cell can be calculated. Using an automated flow control system, the same cell can be flowed repeatedly (every few seconds) through the measurement channel, so that the mass of this single cell can typically be followed over a few minutes or more. The very low noise associated with this measurement allows precise monitoring of the growth rate of a single cell.

By repeating this experiment with a large number of single cells, each with a naturally variable size, Godin et al. ${ }^{1}$ demonstrated that cell growth rate is proportional to cell mass, thus strengthening the exponential growth assumption stated above and confirming previous data using indirect fluorescence markers ${ }^{2}$. The single-cell mass doubling time obtained from these data is in good agreement with the ones obtained for cells growing in a test tube over several generations, a finding that corroborates the necessity for a coupling between cell growth and division. Interestingly, cells with a given size displayed large cell-tocell growth rate variability that is not attributable to experimental errors. Knowing that a large fraction of cell resources is dedicated to mass biogenesis and that growth involves a huge number of molecules (RNAs, ribosomes and others), it is likely that this variability is not related to the stochastic nature of molecular events but rather has an extrinsic origin, which is yet to be determined.

This technique ${ }^{1}$ has several advantages over methods that have been used in previous studies to assay cell growth in single cells: cell mass is a better indicator of protein content than volume. This is especially true in budding yeast, in which the vacuoles can have varying size that greatly affects cell volume but does not contribute much to dry mass. In addition, this label-free technique, provided it will be combined with optical detection in the future, will afford a unique opportunity to characterize cell growth-related processes both at the cellular (measuring cell mass) and molecular (using fluorescent reporters) level. Coupled with classical genetic approaches, this should add to our understanding of the coordination between cell growth and division $^{6}$. From a technical point of view, this microfluidics-based device could be very useful for growth rate-driven cell-sorting applications. Lastly, future applications might include monitoring the response of a given cell's instantaneous growth rate to a sudden change in its environment (for example, heat or high-osmolarity shock) and therefore help refine our understanding of the effects of the canonical signal transduction pathways on cell growth with single-cell resolution.

\section{COMPETING FINANCIAL INTERESTS}

The author declares no competing financial interests.

1. Godin, M. et al. Nat. Methods 7, 387-390 (2010).

2. Di Talia, S., Skotheim, J.M., Bean, J.M., Siggia, E.D. \& Cross, F.R. Nature 448, 947-951 (2007).

3. Elliott, S.G. \& McLaughlin, C.S. Proc. Natl. Acad. Sci. USA 75, 4384-4388 (1978).

4. Goranov, A.I. et al. Genes Dev. 23, 1408-1422 (2009).

5. Burg, T.P. et al. Nature 446, 1066-1069 (2007).

6. Jorgensen, P., Nishikawa, J.L., Breitkreutz, B.J. \& Tyers, M. Science 297, 395-400 (2002). 